\title{
New approach to condensed pyrid-2-ones
}

\author{
Eva Maria Brun, Salvador Gil, and Margarita Parra*
}

Department of Organic Chemistry, Ed. Investigación, University of Valencia, Dr. Moliner, 50, 46100 Burjassot, Valencia, SPAIN.

E-mail: Margarita.Parra@uv.es

(received 01 Dec 2002; accepted 14 Jan 2003; published on the web 22 Jan 2003)

\begin{abstract}
We wish to report a simple procedure for the preparation of 5-substituted-thienopyridin-7-ones and 7-substituted-1,6-naphthyridin-5(6H)-ones, in good yields, from the dianions of 3methylthiophene-2-carboxylic and 2-methylnicotinic acids on treatment with nitriles.
\end{abstract}

Keywords: Carboxylic acids, nitriles, addition reactions, heterocycles, tandem reactions

\section{Introduction}

Development of new approaches to the 2-pyridone ring is a topical area of continuous interest due to the high number of biologically active molecules containing this moiety ${ }^{1}$ and to the facile conversion of pyridones to the corresponding pyridines. ${ }^{2}$ Tautomerism between 2-pyridones and 2-hydroxypyridines receives constant attention because these compounds may act as simple models for investigating the mechanisms of some enzymatic reactions or for discerning the behaviour of nucleic acids bases in connection with mutation due to base mispairing. ${ }^{3}$ Recent studies have shown the usefulness of 2-pyridones as intermolecular connectors between building blocks in material science. ${ }^{4}$ Thus, despite the large number of methods known for their synthesis, ${ }^{5}$ new procedures are continuously being developed. ${ }^{6}$

Reactions of aryl amide enolates with nitriles to afford 2-isoquinolines are known, ${ }^{7}$ but our studies on the reactivity of dienediolates from unsaturated carboxylic acids had led us to a simpler synthesis of 4,6-disubstituted- and 3,4,6-trisubstituted-2-pyridones from easily accessible carboxylic acids and nitriles. ${ }^{8}$ On work-up, pure pyridone was isolated from the neutral fraction whereas the starting acid was recovered from the acidic fraction. The method has been readily reproduced showing it to be reliable. ${ }^{9}$ We found it interesting to study its extension to the synthesis of condensed heteroaromatic systems, both by introduction of heterocyclic nitriles and by using the well known o-toluic (2), 3-methyl-2-thiophenecarboxylic (3) and 2-methylnicotinic (4) acids that, after double deprotonation afford the corresponding lithium dianions. The synthetic interest of the dianion from $o$-toluic acid (2) has been amply demonstrated ${ }^{10}$ but, 
surprisingly, the dianions from 3-methyl-2-thiophenecarboxylic (3) and 2-methylnicotinic (4) acids have received little study. ${ }^{11}$

1<smiles>CC(C)=CC(=O)O</smiles>

2<smiles>Cc1ccccc1C(=O)O</smiles>

3<smiles>Cc1ccsc1C(=O)O</smiles>

4<smiles>Cc1ncccc1C(=O)O</smiles>

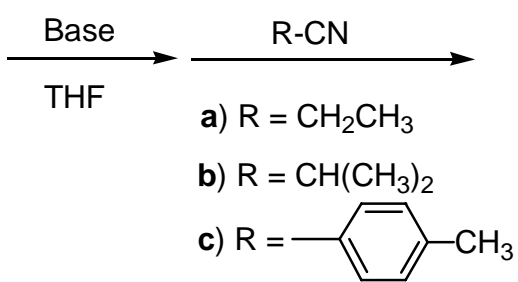

d) $\mathrm{R}$<smiles>[R]Cc1ccccc1-c1ccccc1</smiles>

5<smiles>[R]C=CCCC</smiles>

8

\section{Scheme 1}

\section{Results and Discussion}

The results obtained on reaction of these dianions and nitriles (Scheme 1) are summarized in Table 1.

Although reaction conditions are not critical to obtain the corresponding pyridones, yields are variable and optimisation for each acid and nitrile was performed, specially those leading to 5substituted-thienopyridin-7(6H)-ones (7) and 7-substituted-1,6-naphthyridin-5(6H)-ones (8) as they render information about the generation and behaviour of 3-methyl-2-thiophenecarboxylic (3) and 2-methylnicotinic (4) dianions. Room temperature, 24 hours of reaction time and 1:1 ratio of acid and nitrile was always the best choice. On the other hand, the nature and amount of the amine used for acid deprotonation determines the reaction yield. In most cases, dienediolates of unsaturated carboxylic acids can be generated, without Barbier's reduction or Michael adduct formation, by deprotonation of the corresponding acid with butyllithium in the presence of a catalytic amount of amine. ${ }^{12}$ This renders dienediolates compatible with a large number of functional groups, as it happens with nitriles where self-condensation is minimized under these conditions. Unfortunately, this cannot be considered a general rule (see entries 6 to 9 in Table 1) and it is convenient to optimise the amine and its amount for each acid and nitrile, as used to happen in similar reactions. ${ }^{7}$ 


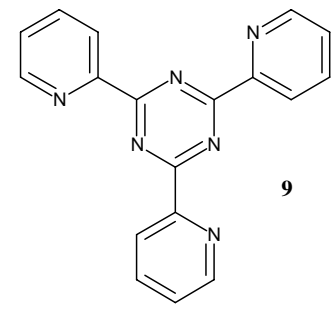

\section{Figure 1}

2-Pyridinecarbonitrile (e) is specially prone to give self-condensation under the basic conditions used, leading to complicated reaction mixtures until the right conditions, usually catalytic amount of amine, were found. In some cases (entry 12) only pure trimerization product, 2,4,6-tri(2-pyridyl)-1,3,5-triazine (9), could be obtained in a good yield (Figure 1).

Table 1. Nucleophilic addition to nitriles

\begin{tabular}{|c|c|c|c|c|c|}
\hline Entry & Acid & Nitrile & $\begin{array}{l}\text { BuLi (2eq) + } \\
\text { Amine (n eq) }\end{array}$ & $\begin{array}{c}\text { Recovered } \\
\text { Acid (\%) }\end{array}$ & $\begin{array}{c}\text { Product } \\
\text { (Yield, \%) }\end{array}$ \\
\hline 1 & 1 & e & $\mathrm{Et}_{2} \mathrm{NH}(2)$ & 4 & 5e (40) \\
\hline 2 & 1 & e & $\mathrm{i}-\mathrm{Pr}_{2} \mathrm{NH}(0.4)$ & 51 & 5e (22) \\
\hline 3 & 2 & e & $\mathrm{Et}_{2} \mathrm{NH}(2)$ & $70^{\mathrm{a}}$ & \\
\hline 4 & 2 & e & $\mathrm{i}-\mathrm{Pr}_{2} \mathrm{NH}(0.4)$ & 30 & 6e (62) \\
\hline 5 & 3 & $\mathrm{a}$ & $\mathrm{i}-\mathrm{Pr}_{2} \mathrm{NH}(0.4)$ & 25 & 7a (56) \\
\hline 6 & 3 & $\mathrm{~b}$ & $\mathrm{Et}_{2} \mathrm{NH}(2)$ & 71 & $7 b(25)$ \\
\hline 7 & 3 & $\mathrm{~b}$ & $\mathrm{Et}_{2} \mathrm{NH}(0.4)$ & 82 & $7 b(16)$ \\
\hline 8 & 3 & $\mathrm{~b}$ & $\mathrm{i}-\mathrm{Pr}_{2} \mathrm{NH}(2)$ & 47 & $7 b(41)$ \\
\hline 9 & 3 & $\mathrm{~b}$ & $\mathrm{i}-\mathrm{Pr}_{2} \mathrm{NH}(0.4)$ & 34 & $7 b(62)$ \\
\hline 10 & 3 & c & $\mathrm{i}-\mathrm{Pr}_{2} \mathrm{NH}(0.4)$ & 40 & 7c (55) \\
\hline 11 & 3 & d & $\mathrm{i}-\mathrm{Pr}_{2} \mathrm{NH}(0.4)$ & 33 & $7 d(62)$ \\
\hline 12 & 3 & e & $\mathrm{i}-\mathrm{Pr}_{2} \mathrm{NH}(0.4)$ & $65^{b}$ & \\
\hline 13 & 4 & $\mathrm{a}$ & $\mathrm{Et}_{2} \mathrm{NH}(2)$ & 7 & $8 a(35)$ \\
\hline 14 & 4 & $\mathrm{a}$ & $\mathrm{Et}_{2} \mathrm{NH}(0.4)$ & 5 & 8a (32) \\
\hline 15 & 4 & $\mathrm{~b}$ & $\mathrm{Et}_{2} \mathrm{NH}(2)$ & 6 & $8 b(50)$ \\
\hline 16 & 4 & b & $\mathrm{Et}_{2} \mathrm{NH}(0.4)$ & 5 & $8 b(21)$ \\
\hline 17 & 4 & $\mathrm{~b}$ & $\mathrm{i}-\mathrm{Pr}_{2} \mathrm{NH}(0.4)$ & 10 & $8 b(25)$ \\
\hline 18 & 4 & c & $\mathrm{Et}_{2} \mathrm{NH}(2)$ & & $8 c(42)$ \\
\hline 19 & 4 & c & $\mathrm{Et}_{2} \mathrm{NH}(0.4)$ & 9 & 8c (61) \\
\hline 20 & 4 & d & $\mathrm{Et}_{2} \mathrm{NH}(2)$ & 8 & 8d (75) \\
\hline 21 & 4 & d & $\mathrm{Et}_{2} \mathrm{NH}(0.4)$ & 4 & 8d (64) \\
\hline 22 & 4 & $d$ & $\mathrm{i}-\mathrm{Pr}_{2} \mathrm{NH}(0.4)$ & & $8 d(0)$ \\
\hline 23 & 4 & $\mathrm{e}$ & $\mathrm{i}-\mathrm{Pr}_{2} \mathrm{NH}(0.4)$ & & $8 e(56)$ \\
\hline
\end{tabular}

a.- 47\% polymeric material; ${ }^{\mathrm{b}}$.- 46\% 2,4,6-tri(pyridyl)-1,3,5-triazine (9). 


\section{Tautomerism}

Both amidic and phenolic tautomeric forms of compound 6e precipitate from water in a 2:1 ratio. By treatment with chloroform, both tautomers can be easily isolated. The first is soluble in chloroform and the second is highly insoluble. Their spectroscopic data are consistent with the proposed structures for both tautomers and provide us with a model that allows us to establish the amide form as the major tautomer in the rest of the compounds described.

\section{Conclusions}

In conclusion it can be said that o-methylarenic acids may be converted, under suitable conditions, into the corresponding dianions which show similar nucleophilic behaviour to the dienediolates of unsaturated carboxylic acids. It has been shown that condensed heteroaromatic systems are obtained from these dianions and nitriles through a tandem addition-cyclization process. On work up, pure products are easily isolated from any remaining starting material.

\section{Experimental Section}

General Procedures. M.p.s. were determined with a Reichert apparatus and are uncorrected. IR spectral data were obtained as liquid films or $\mathrm{KBr}$ discs using a Perkin-Elmer 281 spectrophotometer. NMR spectra were recorded in $\mathrm{CDCl}_{3}$ or deuterated DMSO solutions, using a Varian Unity 300 or Unity 400 spectrometer. A series of COSY, NOESY and HMQC nmr experiments were carried out to assign both ${ }^{1} \mathrm{H}$ and ${ }^{13} \mathrm{C}$ signals. Elemental analyses were determined by "Servicio de Semimicroanálisis del Centro de Investigación y Desarrollo (CSIC) de Barcelona". High resolution mass spectra were determined with a UG Autoespec spectrometer. Silica gel Merck 60 (230-400 mesh) was used for flash column chromatography, with hexane/diethyl ether mixtures for elution.

All reactions were carried out under argon atmosphere, using standard conditions for exclusion of moisture, in oven dried glassware, in THF freshly distilled from blue benzophenone ketyl and with diethyl amine and diisopropylamine distilled from $\mathrm{CaH}_{2}$. The reaction temperature $\left(-78^{\circ} \mathrm{C}\right)$ was achieved by cooling with a $\mathrm{CO}_{2}$ /acetone bath. Organic extracts were dried over anhyd $\mathrm{MgSO}_{4}$, and solutions were evaporated under reduced pressure with a rotary evaporator and a bath at $40^{\circ} \mathrm{C}$.

\section{General procedure for the synthesis of 2-pyridones}

Carboxylic acid (2.25 mmol) in THF (2 ml) was slowly added to a stirred mixture of $\mathrm{n}$ butyllithium and dialkylamine (see Table 1$)$ in THF $(2 \mathrm{ml})$ at $-78^{\circ} \mathrm{C}$, according to the method already described. The solution was stirred for $60 \mathrm{~min}$ at $0^{\circ} \mathrm{C}$ and cooled again at $-78^{\circ} \mathrm{C}$. Nitrile (2.25 mmol) in THF (2 ml) was added dropwise, and the solution stirred at room temperature for 
24 hours. Water $(20 \mathrm{ml})$ was added and the product isolated by crystallization from crude in most cases, leading to pure products. In other cases, water was added, extracted with dichloromethane $(3 \times 15 \mathrm{ml})$ and the combined organic layers were washed with brine to neutral $\mathrm{pH}$, and dried. Evaporation of the solvent gave crude 2-pyridone product. The aqueous layer was acidified under ice-cooling bath by careful addition of conc. hydrochloric acid and then extracted with ethyl acetate ( $3 \times 15 \mathrm{ml})$. The acidic organic layer was washed with brine, dried and evaporation of the solvent gave the recovered starting acid.

4-Methyl-6-(2-pyridinyl)pyridin-2(1H)-one (5e). From 3-methyl-2-butenoic acid 1 (226 mg, $2.25 \mathrm{mmol}$ ) and 2-cyanopyridine e (235 mg, $2.25 \mathrm{mmol}$ ) and lithium diethylamide (from BuLi $3.2 \mathrm{ml}, 1.6 \mathrm{molar}$, and diethylamine $0.5 \mathrm{ml}, 5 \mathrm{mmol}$ ).Yield: $168 \mathrm{mg}(40 \%)$; m.p. 84-85 ${ }^{\circ} \mathrm{C}$ (yellow prism). Recovered starting acid: $9 \mathrm{mg}(4 \%)$.

IR (KBr): $v_{\max }=3400,3100-2800,1655,1614,1580,1481,854 \mathrm{~cm}^{-1}$.

${ }^{1} \mathrm{H}$ NMR (MeOD, 300MHz): $\delta=8.63$ (d, $1 \mathrm{H}, J=4.8 \mathrm{~Hz}, \mathrm{~N}-\mathrm{CH}$ ), 7.92 (d, $1 \mathrm{H}, J=8.1 \mathrm{~Hz}, \mathrm{~N}-\mathrm{C}-$ $\mathrm{C} \underline{\mathrm{H}}$ ), 7.84 (dt, $1 \mathrm{H}, J=8.1$ and $1.5 \mathrm{~Hz}, \mathrm{~N}-\mathrm{CH}-\mathrm{CH}-\mathrm{C} \underline{\mathrm{H}}-\mathrm{CH}$ ), 7.38 (ddd, $1 \mathrm{H}, J=8.1,4.8 \mathrm{and} 1.5 \mathrm{~Hz}$,

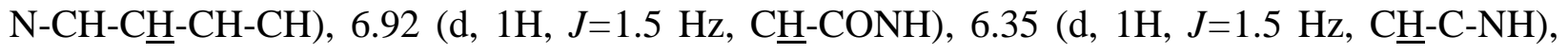
$2.26\left(\mathrm{~s}, 3 \mathrm{H}, \mathrm{CH}_{3}\right)$

${ }^{13} \mathrm{C}$ NMR (MeOD, 75MHz) $\delta=164.1(\mathrm{C}=\mathrm{O}), 154.7\left(\mathrm{C}-\mathrm{CH}_{3}\right), 149.3(\mathrm{~N}-\mathrm{CH}), 148.1$ ( $\left.\underline{\mathrm{C}}-\mathrm{NHCO}\right)$,

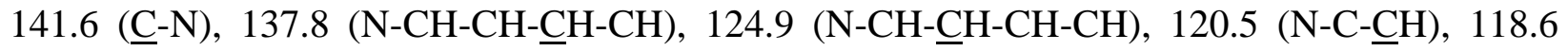
( $\underline{\mathrm{C}} \mathrm{H}-\mathrm{CONH}), 107.4$ (ㅡㅐ-C-NHCO), $20.6\left(\mathrm{CH}_{3}\right)$.

MS (EI): $m / z(\%)=186\left(\mathrm{M}^{+}, 100\right), 158\left(\mathrm{M}^{+}-\mathrm{CO}, 41\right), 157\left(\mathrm{M}^{+}-\mathrm{COH}, 56\right), 79\left(\mathrm{C}_{5} \mathrm{H}_{5} \mathrm{~N}^{+}, 10\right), 78$ $\left(\mathrm{C}_{5} \mathrm{H}_{4} \mathrm{~N}^{+}, 8\right)$

HRMS: $\mathrm{m} / \mathrm{z}$ calcd for $\mathrm{C}_{11} \mathrm{H}_{10} \mathrm{~N}_{2} \mathrm{O}\left(\mathrm{M}^{+}\right)$186.0793, found: 186.0792 .

3-(2-Pyridinyl)isoquinolin-1(2H)-one (6e). From o-toluic acid 2 (306 mg, $2.25 \mathrm{mmol}$ ) and 2cyanopyridine e (237 mg, $2.25 \mathrm{mmol}$ ) and lithium diisopropylamide (from BuLi $3.2 \mathrm{ml}, 1.6$ molar, and isopropylamine $0.14 \mathrm{ml}, 0.96 \mathrm{mmol}$ ).Yield: $310 \mathrm{mg}$ (62\%). Recovered starting acid: $92 \mathrm{mg}(30 \%)$.

The fraction soluble in $\mathrm{CHCl}_{3}$ was identified as the above isoquinolone, m.p. $136-137^{\circ} \mathrm{C}$ (yellow solid). IR (KBr): $v_{\max }=3329,3200-2900,1659,1470,1437,1342,1146,855,788,730 \mathrm{~cm}^{-1} .{ }^{1} \mathrm{H}$ NMR $\left(\mathrm{CDCl}_{3}, 500 \mathrm{MHz}\right): \delta=10.44$ (brs, $\left.1 \mathrm{H}, \mathrm{NH}\right), 8.66$ (d, $\left.1 \mathrm{H}, J=4.1 \mathrm{~Hz}, \mathrm{C} \underline{\mathrm{H}}-\mathrm{N}\right), 8.47$ (d, $1 \mathrm{H}$, $J=8.0 \mathrm{~Hz}, \mathrm{C} \underline{\mathrm{H}}-\mathrm{C}=\mathrm{C}-\mathrm{CONH}$ ), 7.94 (d, $1 \mathrm{H}, J=7.5 \mathrm{~Hz}, \mathrm{~N}-\mathrm{C}-\mathrm{C} \underline{\mathrm{H}}$ ), 7.82 (dt, $1 \mathrm{H}, J=7.5$ and $1.5 \mathrm{~Hz}$, N-CH-CH-CH-CH), 7.69 (dt, $1 \mathrm{H}, J=8.0$ and $1.3 \mathrm{~Hz}, \mathrm{C} \underline{\mathrm{H}}-\mathrm{CH}-\mathrm{C}-\mathrm{CONH}$ ), 7.65 (d, $1 \mathrm{H}, J=8.0 \mathrm{~Hz}$, C $\underline{H}-\mathrm{C}-\mathrm{CONH}$ ), 7.53 (dt, $1 \mathrm{H}, J=8.0$ and $1.3 \mathrm{~Hz}, \mathrm{C} \underline{\mathrm{H}}-\mathrm{CH}-\mathrm{C}-\mathrm{C}-\mathrm{CONH}$ ), 7.35 (ddd, 1H, $J=7.5,4.1$ and $1.5 \mathrm{~Hz}, \mathrm{~N}-\mathrm{CH}-\mathrm{C} \underline{\mathrm{H}}-\mathrm{CH}-\mathrm{CH}), 7.16$ (s, $1 \mathrm{H}, \mathrm{C} \underline{\mathrm{H}}-\mathrm{C}-\mathrm{NH}) .{ }^{13} \mathrm{C}$ NMR (MeOD, 75MHz) $\delta=162.3$

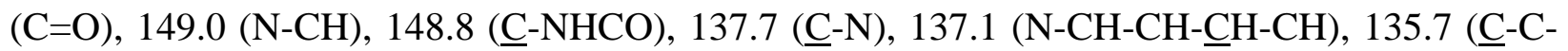

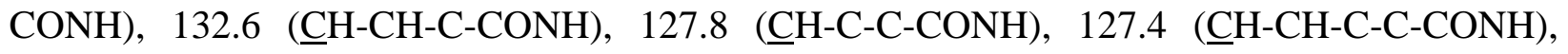

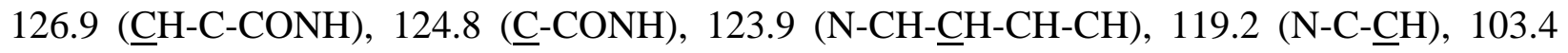
(ㅡ-C-NHCO). MS (EI): m/z (\%) = $223\left(\mathrm{M}^{+}+1,18\right), 222\left(\mathrm{M}^{+}, 100\right), 194\left(\mathrm{M}^{+}-\mathrm{CO}, 14\right), 193\left(\mathrm{M}^{+}-\right.$ $\mathrm{COH}, 8), 118\left(\mathrm{C}_{8} \mathrm{H}_{6} \mathrm{O}^{+}, 10\right), 78\left(\mathrm{C}_{5} \mathrm{H}_{4} \mathrm{~N}^{+}, 5\right)$ HRMS: $m / z$ calcd for $\mathrm{C}_{11} \mathrm{H}_{10} \mathrm{~N}_{2} \mathrm{O}\left(\mathrm{M}^{+}\right)$222.0793, found: 222.0796. 
The fraction soluble in $\mathrm{MeOH}$ was identified as the hydroxyisoquinoline, m.p. $180-82^{\circ} \mathrm{C}$ (dec.) (yellow solid). IR (KBr): $v_{\max }=3173,3050-2900,1639,1473,1435,1343,864,788,786 \mathrm{~cm}^{-1}$. ${ }^{1} \mathrm{H}$ NMR (MeOD, 500MHz): $\delta=8.64$ (d, $1 \mathrm{H}, J=4.8 \mathrm{~Hz}, \mathrm{C} \underline{\mathrm{H}}-\mathrm{N}$ ), 8.29 (d, $1 \mathrm{H}, J=7.8 \mathrm{~Hz}, \mathrm{C} \underline{\mathrm{H}}-\mathrm{C}-\mathrm{C}-$ CONH), 8.09 (dt, $1 \mathrm{H}, J=8.0$ and $0.9 \mathrm{~Hz}, \mathrm{~N}-\mathrm{C}-\mathrm{C} \underline{\mathrm{H}}), 7.89$ (dt, $1 \mathrm{H}, J=8.0$ and $1.8 \mathrm{~Hz}, \mathrm{~N}-\mathrm{CH}-\mathrm{CH}-$ $\mathrm{C} \underline{\mathrm{H}}-\mathrm{CH}$ ), 7.74 (m, 2H, C$-\mathrm{H}-\mathrm{CH}-\mathrm{C}-\mathrm{CONH}$ and $\mathrm{C} \underline{\mathrm{H}}-\mathrm{C}-\mathrm{CONH}$ ), 7.52 (ddd, $1 \mathrm{H}, J=7.8,6.0$ and 2.4 $\mathrm{Hz}, \mathrm{C} \underline{\mathrm{H}}-\mathrm{CH}-\mathrm{C}-\mathrm{C}-\mathrm{CONH}$ ), 7.44 (s, $1 \mathrm{H}, \mathrm{C} \underline{\mathrm{H}}-\mathrm{C}-\mathrm{NH}$ ), 7.38 (ddd, $1 \mathrm{H}, J=8.0,4.8$ and $0.9 \mathrm{~Hz}, \mathrm{~N}-\mathrm{CH}-$ C $\underline{H}-\mathrm{CH}-\mathrm{CH}) .{ }^{13} \mathrm{C}$ NMR (MeOD, 75MHz) $\delta=163.6(\mathrm{COH}), 150.5$ (C-NHCO), $149.0(\mathrm{~N}-\mathrm{CH})$,

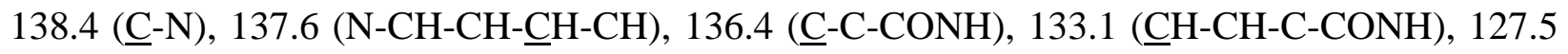

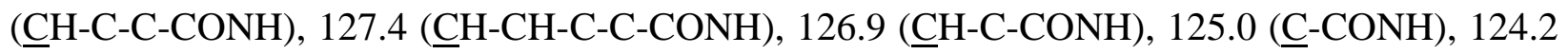
(N-CH-ChH-CH-CH), 120.0 (N-C-CH), 104.8 (ㅡㅐ-C-NHCO).

5-Ethyl-6,7-dihydrothieno[2,3-c]pyridin-7-one (7a). From 3-methyl-2-thiophenecarboxylic acid 3 (326 mg, $2.25 \mathrm{mmol}$ ) and propionitrile a $(0.16 \mathrm{ml}, 2.25 \mathrm{mmol}$ ) and lithium diisopropylamide (from BuLi $3.2 \mathrm{ml}, 1.6$ molar, and isopropylamine $0.14 \mathrm{ml}, 0.96 \mathrm{mmol}$ ).Yield: $228 \mathrm{mg}$ (56\%); m.p. 171-172 ${ }^{\circ} \mathrm{C}$ (brown prisms). Recovered starting acid: $82 \mathrm{mg}(25 \%) . \mathrm{IR}(\mathrm{KBr})$ : $v_{\max }=3420,3100-2800,1801,1636,1525,1474,1048,825 \mathrm{~cm}^{-1} .{ }^{1} \mathrm{H}$ NMR $\left(\mathrm{CDCl}_{3}, 300 \mathrm{MHz}\right)$ : $\delta=10.58$ (s, $1 \mathrm{H}, \mathrm{NH}), 7.70$ (d, $1 \mathrm{H}, J=5.1 \mathrm{~Hz}, \mathrm{C} \underline{\mathrm{H}}=\mathrm{CH}-\mathrm{S}$ ), 7.18 (d, $1 \mathrm{H}, J=5.1 \mathrm{~Hz}, \mathrm{CH}=\mathrm{C} \underline{H}-\mathrm{S}$ ), 6.50 (s, $1 \mathrm{H}, \mathrm{C} \underline{\mathrm{H}}-\mathrm{C}-\mathrm{NH}), 2.70$ (q, $2 \mathrm{H}, J=7.5 \mathrm{~Hz}, \underline{\mathrm{C}}_{2}-\mathrm{CH}_{3}$ ), 1.3 (t, $\left.3 \mathrm{H}, J=7.5 \mathrm{~Hz}, \mathrm{CH}_{2}-\mathrm{CH}_{3}\right) .{ }^{13} \mathrm{C}$ NMR $\left(\mathrm{CDCl}_{3}, 75 \mathrm{MHz}\right) \delta=160.8(\mathrm{C}=\mathrm{O}), 147.5(\mathrm{CH}=\underline{\mathrm{C}}-\mathrm{NH}), 145.5$ ( $\left.\underline{\mathrm{C}}=\mathrm{C}-\mathrm{S}\right), 133.5$ (CH=$\left.-\underline{\mathrm{CH}}-\mathrm{S}\right)$, 126.8 (C=-ㅡ), 124.2 ( $\underline{\mathrm{CH}}=\mathrm{CH}-\mathrm{S}), 100.7$ ( $\underline{\mathrm{CH}}-\mathrm{C}-\mathrm{NH}), 26.3\left(\underline{\mathrm{CH}}_{2}-\mathrm{CH}_{3}\right), 12.8\left(\underline{\mathrm{C}} \mathrm{H}_{3}\right) . \mathrm{MS}$ (EI): $\mathrm{m} / \mathrm{z}(\%)=179\left(\mathrm{M}^{+}, 100\right), 178\left(\mathrm{M}^{+}-\mathrm{H}, 68\right), 164\left(\mathrm{M}^{+}-\mathrm{CH}_{3}, 38\right), 160\left(\mathrm{M}^{+}-\mathrm{H}-\mathrm{H}_{2} \mathrm{O}, 5\right), 151\left(\mathrm{M}^{+}-\mathrm{CO}\right.$, 12), $137\left(\mathrm{M}^{+}-\mathrm{CON}, 19\right), 95\left(\mathrm{C}_{5} \mathrm{H}_{3} \mathrm{~S}^{+}\right.$, 5). HRMS: $\mathrm{m} / z$ calcd for $\mathrm{C}_{9} \mathrm{H}_{9} \mathrm{NOS}\left(\mathrm{M}^{+}\right)$179.0405, found: 179.0405 .

5-Isopropyl-6,7-dihydrothieno[2,3-c]pyridin-7-one (7b). From 3-methyl-2-thiophene carboxylic acid 3 (326 mg, $2.25 \mathrm{mmol}$ ) and isobutyronitrile b $(0.20 \mathrm{ml}, 2.25 \mathrm{mmol}$ ) and lithium diisopropylamide (from BuLi $3.2 \mathrm{ml}, 1.6$ molar, and isopropylamine $0.14 \mathrm{ml}, 0.96 \mathrm{mmol}$ ).Yield: 276 mg (62\%); m.p. 164-165 ${ }^{\circ} \mathrm{C}$ (orange prisms). Recovered starting acid: $117 \mathrm{mg}$ (34\%). IR $(\mathrm{KBr}): v_{\max }=3295 ; 3142,1646,1626$ 1530,1482, 1087, 1050, 915, $818 \mathrm{~cm}^{-1} .{ }^{1} \mathrm{H} \mathrm{NMR}\left(\mathrm{CDCl}_{3}\right.$, 300MHz): $\delta=10.90$ (s, $1 \mathrm{H}, \mathrm{NH}$ ), 7.68 (d, $1 \mathrm{H}, J=5.1 \mathrm{~Hz}, \mathrm{~S}-\mathrm{C} \underline{\mathrm{H}}=\mathrm{CH}$ ), 7.20 (d, $1 \mathrm{H}, J=5.1 \mathrm{~Hz}, \mathrm{~S}-$ $\mathrm{CH}=\mathrm{C} \underline{\mathrm{H}}), 6.50$ (s, $1 \mathrm{H}, \mathrm{C}-\mathrm{C} \underline{\mathrm{H}}-\mathrm{C}-\mathrm{NH}), 2.95$ [m, 1H, C-C $\left.\underline{\mathrm{H}}\left(\mathrm{CH}_{3}\right)_{2}\right], 1.35$ [d, 6H, J=6.3 Hz, C$\left.\mathrm{CH}\left(\mathrm{CH}_{3}\right)_{2}\right] .{ }^{13} \mathrm{C}$ NMR $\left(\mathrm{CDCl}_{3}, 75 \mathrm{MHz}\right) \delta=160.7(\mathrm{C}=\mathrm{O}), 149.7(\underline{\mathrm{C}}-\mathrm{NH}), 147.4(\mathrm{CH}-\underline{\mathrm{C}}=\mathrm{C}-\mathrm{S})$ $133.4(\underline{\mathrm{CH}}=\mathrm{CH}-\mathrm{S}), 127.8(\mathrm{~S}-\underline{\mathrm{C}}=\mathrm{C}), 124.3(\mathrm{CH}=\underline{\mathrm{CH}}-\mathrm{S}), 99.0(\underline{\mathrm{CH}}=\mathrm{C}-\mathrm{NH}), 32.1\left[\underline{\mathrm{CH}}\left(\mathrm{CH}_{3}\right)_{2}\right], 21.8$ [CH$\left.\left(\mathrm{CH}_{3}\right)_{2}\right]$. MS (EI): $\mathrm{m} / \mathrm{z}(\%)=193\left(\mathrm{M}^{+}, 81\right), 178\left(\mathrm{M}^{+}-\mathrm{CH}_{3}, 100\right) 165\left(\mathrm{M}^{+}-\mathrm{CO}, 10\right) 160\left(\mathrm{M}^{+}-\right.$ $\mathrm{CH}_{3}-\mathrm{H}_{2} \mathrm{O}$, 19). HRMS: $\mathrm{m} / \mathrm{z}$ calcd for $\mathrm{C}_{10} \mathrm{H}_{11} \mathrm{NOS}\left(\mathrm{M}^{+}\right)$193.0561, found: 193.0565 .

5-(4-Methylphenyl)-6,7-dihydrothieno[2,3-c]pyridin-7-one (7c) From 3-methyl-2thiophenecarboxylic acid 3 (326 mg, $2.25 \mathrm{mmol}$ ) and p-methylbenzonitrile c (266 mg, $2.25 \mathrm{mmol}$ ) and lithium diisopropylamide (from BuLi $3.2 \mathrm{ml}, 1.6$ molar, and isopropylamine $0.14 \mathrm{ml}, 0.96 \mathrm{mmol}$ ).Yield: $301 \mathrm{mg}$ (55\%); m.p. $252-254^{\circ} \mathrm{C}$ (yellow solid). Recovered starting acid: $131 \mathrm{mg}(40 \%)$. IR (KBr): $v_{\max }=3100-2900,1630,1513,1474,1186,1132,1045,808 \mathrm{~cm}^{-1}$. ${ }^{1} \mathrm{H}$ NMR $\left(\mathrm{CDCl}_{3}, 300 \mathrm{MHz}\right): \delta=9.38(\mathrm{~s}, 1 \mathrm{H}, \mathrm{NH}), 7.73(\mathrm{~d}, 1 \mathrm{H}, J=5.1 \mathrm{~Hz}, \mathrm{CH}=\mathrm{CH}-\mathrm{S}), 7.58$ (d, 2H, $J=8.1 \mathrm{~Hz}, 2 \mathrm{Ar}-\mathrm{H}$ ), 7.30 (d, 2H, $J=8.1 \mathrm{~Hz}, 2 \mathrm{Ar}-\mathrm{H}$ ), 7.27 (d, $1 \mathrm{H}, J=5.1 \mathrm{~Hz}, \mathrm{CH}=\mathrm{C} \underline{\mathrm{H}}-\mathrm{S}$ ), 6.9 
(s, 1H, Cㅍ-C-NH), 2.42 (s, 3H, $\left.\mathrm{CH}_{3}\right) .{ }^{13} \mathrm{C} \mathrm{NMR}\left(\mathrm{CDCl}_{3}, 300 \mathrm{MHz}\right) \delta=160.2(\mathrm{C}=\mathrm{O}), 147.3(\mathrm{CH}-$

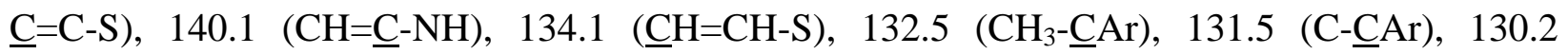
(2CHAr), 126.3 (2CHAr), 124.9 (CH=ㅡ-S), 101.3 ( $\underline{\mathrm{CH}}-\mathrm{C}-\mathrm{NH}), 21.5\left(\mathrm{CH}_{3}\right) . \mathrm{MS}(\mathrm{EI}): \mathrm{m} / \mathrm{z}(\%)$ $=241\left(\mathrm{M}^{+}, 100\right), 226\left(\mathrm{M}^{+}-\mathrm{CH}_{3}, 4\right), 119\left(\mathrm{M}^{+}-\mathrm{C}_{8} \mathrm{H}_{7} \mathrm{O}, 90\right), 91\left(\mathrm{C}_{7} \mathrm{H}_{7}^{+}, 25\right)$. HRMS: $\mathrm{m} / \mathrm{z}$ calcd for $\mathrm{C}_{14} \mathrm{H}_{11} \mathrm{NOS}\left(\mathrm{M}^{+}\right)$241.0561, found 241.0554.

5-(4-Chlorophenyl)-6,7-dihydrothieno[2,3-c]pyridin-7-one (7d). From 3-methyl-2thiophenecarboxylic acid 3 (326 mg, $2.25 \mathrm{mmol}$ ) and p-chlorobenzonitrile d (310 mg, $2.25 \mathrm{mmol}$ ) and lithium diisopropylamide (from BuLi $3.2 \mathrm{ml}, 1.6$ molar, and isopropylamine $0.14 \mathrm{ml}, 0.96 \mathrm{mmol})$.Yield: $373 \mathrm{mg}$ (62\%); m.p. 281-283 ${ }^{\circ} \mathrm{C}$ (brown solid). Recovered starting acid: $106 \mathrm{mg}$ (33\%). IR (KBr): $v_{\max }=3142,3052,2913,1662,1615,1499,1422,1137,1090$, 1014, $807 \mathrm{~cm}^{-1} .{ }^{1} \mathrm{H} \mathrm{NMR}\left(\mathrm{CDCl}_{3}, 300 \mathrm{MHz}\right): \delta=8.67(\mathrm{~s}, 1 \mathrm{H}, \mathrm{NH}), 7.76(\mathrm{~d}, 1 \mathrm{H}, J=5.1 \mathrm{~Hz}$, $\mathrm{C} \underline{\mathrm{H}}=\mathrm{CH}-\mathrm{S}), 7.62$ (d, 2H, J=8.7 Hz, 2Ar-H), 7.48 (d, 2H, J=8.7 Hz, 2Ar-H), 7.28 (d, 1H,

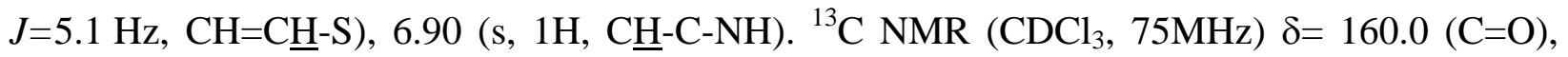
146.8 (CH- $\underline{C}=\mathrm{C}-\mathrm{S}), 142.1$ (CAr), 140.1 (CH=C-NH), 134.1 (2CHAr), 131.5 (Cl-CAr), 130.8

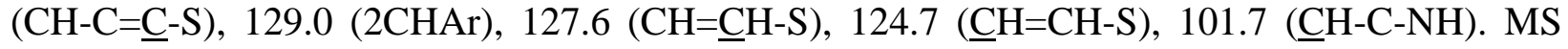
(EI): $m / z(\%)=263\left({ }^{37} \mathrm{Cl} \mathrm{M}^{+}, 40\right) 261\left({ }^{35} \mathrm{ClM}^{+}, 100\right), 226\left(\mathrm{M}^{+}-\mathrm{Cl}, 5\right), 149\left(\mathrm{M}-\mathrm{C}_{6} \mathrm{H}_{5} \mathrm{Cl}, 13\right)$. HRMS: $\mathrm{m} / \mathrm{z}$ calcd for $\mathrm{C}_{13} \mathrm{H}_{8} \mathrm{NOS}^{37} \mathrm{Cl}\left(\mathrm{M}^{+}\right)$262.9986, found: 262.9989. $\mathrm{m} / \mathrm{z}$ calcd for $\mathrm{C}_{13} \mathrm{H}_{8} \mathrm{NOS}^{35} \mathrm{Cl}\left(\mathrm{M}^{+}\right)$261.0015, found: 261.0022.

7-Ethyl-5,6-dihydro[1,6]naphthyridin-5-one (8a). From 2-methylnicotinic acid 4 (307 mg, $2.25 \mathrm{mmol})$ and propionitrile a $(0.16 \mathrm{ml}, 2.25 \mathrm{mmol})$ and lithium diethylamide (from BuLi $3.2 \mathrm{ml}, 1.6$ molar, and diethylamine $0.10 \mathrm{ml}, 0.96 \mathrm{mmol}$ ).Yield: $137 \mathrm{mg}$ (35\%); m.p. 197-198 ${ }^{\circ} \mathrm{C}$ (orange prisms). Recovered starting acid: $15 \mathrm{mg}$ (7\%). IR (KBr): $v_{\max }=3162,3005,2965,1671$, 1636, 1590, 1552, 1466, 1323, 1101, 1073, 903, 860, $772 \mathrm{~cm}^{-1} .{ }^{1} \mathrm{H} \mathrm{NMR}\left(\mathrm{CDCl}_{3}, 300 \mathrm{MHz}\right): \delta=$ 11.70 (s, 1H, NH), 8.87 (d, $1 \mathrm{H}, J=5.1 \mathrm{~Hz}, \mathrm{~N}=\mathrm{CH}-\mathrm{CH}=\mathrm{CH}), 8.62$ (d, 1H, J= $8.2 \mathrm{~Hz}, \mathrm{~N}=\mathrm{CH}-$ $\mathrm{CH}=\mathrm{C} \underline{\mathrm{H}}$ ), 7.32 (dd, $1 \mathrm{H}, J=8.2$ and $5.1 \mathrm{~Hz}, \mathrm{~N}=\mathrm{CH}-\mathrm{C} \underline{\mathrm{H}}=\mathrm{CH}), 6.61$ (s, 1H, $\mathrm{C} \underline{H}=\mathrm{C}-\mathrm{NH}), 2.74$ (q, $\left.2 \mathrm{H}, J=7.5 \mathrm{~Hz}, \underline{\mathrm{CH}}_{2}-\mathrm{CH}_{3}\right), 1.37\left(\mathrm{t}, 3 \mathrm{H}, J=7.5 \mathrm{~Hz}, \mathrm{CH}_{3}\right) \cdot{ }^{13} \mathrm{C} \mathrm{NMR}\left(\mathrm{CDCl}_{3}, 75 \mathrm{MHz}\right) \delta=164.9$ $(\mathrm{C}=\mathrm{O}), \quad 155.1 \quad(\mathrm{~N}-\underline{\mathrm{C}}=\mathrm{C}-\mathrm{CO}), 154.9 \quad(\mathrm{~N}=\underline{\mathrm{CH}}-\mathrm{CH}=\mathrm{CH}), 147.8 \quad(\mathrm{CH}=\underline{\mathrm{C}}-\mathrm{NH}), 135.6 \quad(\mathrm{~N}=\mathrm{CH}-$ $\mathrm{CH}=\underline{\mathrm{CH}}), 120.8(\mathrm{~N}=\mathrm{CH}-\underline{\mathrm{CH}}=\mathrm{CH}), 119.9(\mathrm{~N}-\mathrm{C}=\underline{\mathrm{C}}-\mathrm{CO}), 105.0(\underline{\mathrm{CH}}=\mathrm{C}-\mathrm{NH}), 26.6\left(\underline{\mathrm{CH}}_{2} \mathrm{CH}_{3}\right), 12.2$ $\left(\mathrm{CH}_{3}\right)$. MS (EI): $\mathrm{m} / \mathrm{z}(\%)=174\left(\mathrm{M}^{+}, 100\right), 173\left(\mathrm{M}^{+}-1,69\right), 159\left(\mathrm{M}^{+}-\mathrm{CH}_{3}, 17\right), 146\left(\mathrm{M}^{+}-\mathrm{CO}, 12\right)$, $132\left(\mathrm{M}^{+}-\mathrm{CON}, 11\right)$. HRMS: $\mathrm{m} / \mathrm{z}$ calcd for $\mathrm{C}_{10} \mathrm{H}_{10} \mathrm{~N}_{2} \mathrm{O}\left(\mathrm{M}^{+}\right)$174.0793, found 174.0794.

7-Isopropyl-5,6-dihydro[1,6]naphthyridin-5-one (8b). From 2-methylnicotinic acid 4 (307 mg, $2.25 \mathrm{mmol}$ ) and isobutyronitrile b (0.20 ml, $2.25 \mathrm{mmol})$ and lithium diisopropylamide (from BuLi $3.2 \mathrm{ml}, 1.6$ molar, and diisopropylamine $0.14 \mathrm{ml}, 0.96 \mathrm{mmol}$ ).Yield: $200 \mathrm{mg}$ (50\%); m.p. $190-192^{\circ} \mathrm{C}$ (yellow prisms). Recovered starting acid: $9 \mathrm{mg}(5 \%)$. IR (KBr): $v_{\max }=3163$, 3014, 2962, 2890, 1673, 1636, 1590, 1383, 1246, 979, 903, $857 \mathrm{~cm}^{-1} .{ }^{1} \mathrm{H}$ NMR $\left(\mathrm{CDCl}_{3}\right.$, $300 \mathrm{MHz}): \delta=10.00(\mathrm{~s}, 1 \mathrm{H}, \mathrm{NH}), 8.88(\mathrm{~d}, 1 \mathrm{H}, J=5.1 \mathrm{~Hz}, \mathrm{~N}=\mathrm{C} \underline{\mathrm{H}}), 8.61(\mathrm{~d}, 1 \mathrm{H}, J=8.2 \mathrm{~Hz}$, $\mathrm{N}=\mathrm{CH}-\mathrm{CH}=\mathrm{C} \underline{\mathrm{H}}$ ), 7.35 (dd, $1 \mathrm{H}, J=8.2$ and $5.1 \mathrm{~Hz}, \mathrm{~N}=\mathrm{CH}-\mathrm{C} \underline{H}=\mathrm{CH}), 6.61$ (s, 1H, $\mathrm{C}=\mathrm{C}-\mathrm{NH}$ ), $2.90\left[\mathrm{~m}, 1 \mathrm{H}, \mathrm{C} \underline{\mathrm{H}}\left(\mathrm{CH}_{3}\right)_{2}\right], 1.37\left[\mathrm{~d}, 6 \mathrm{H}, J=5.9 \mathrm{~Hz}, \mathrm{CH}\left(\mathrm{C}_{3}\right)_{2}\right] .{ }^{13} \mathrm{C} \mathrm{NMR}\left(\mathrm{CDCl}_{3}, 75 \mathrm{MHz}\right) \delta=$ $165.0(\mathrm{C}=\mathrm{O}), 155.4$ ( $\underline{\mathrm{C}}=\mathrm{C}-\mathrm{CO}), 155.1(\mathrm{~N}=\underline{\mathrm{CH}}-\mathrm{CH}=\mathrm{CH}), 152.1 \quad(\mathrm{CH}=\underline{\mathrm{C}}-\mathrm{NH}), 135.9(\mathrm{~N}=\mathrm{CH}-$ $\mathrm{CH}=\underline{\mathrm{CH}}), 121.0(\mathrm{~N}=\mathrm{CH}-\underline{\mathrm{CH}}=\mathrm{CH}), 120.4$ (C=ㅡ-CO), $103.8(\underline{\mathrm{CH}}=\mathrm{C}-\mathrm{NH}), 32.6\left[\underline{\mathrm{CH}}\left(\mathrm{CH}_{3}\right)_{2}\right], 21.5$ 
$\left(\mathrm{CH}_{3}\right)$. MS (EI): $\mathrm{m} / \mathrm{z}(\%)=188\left(\mathrm{M}^{+}, 100\right), 187\left(\mathrm{M}^{+}-\mathrm{H}, 19\right), 173\left(\mathrm{M}-\mathrm{CH}_{3}, 99\right), 160(\mathrm{M}-\mathrm{CO}, 12)$, $155\left(\mathrm{M}^{+}-\mathrm{CH}_{3}-\mathrm{H}_{2} \mathrm{O}, 18\right)$. HRMS: $\mathrm{m} / z$ calcd for $\mathrm{C}_{11} \mathrm{H}_{12} \mathrm{~N}_{2} \mathrm{O}\left(\mathrm{M}^{+}\right)$188.0950, found 188.0946 .

7-(4-Methylphenyl)-5,6-dihydro[1,6]naphthyridin-5-one (8c). From 2-methylnicotinic acid 4 (307 mg, $2.25 \mathrm{mmol}$ ) and p-methylbenzonitrile c (269 mg, $2.25 \mathrm{mmol}$ ) and lithium diethylamide (from BuLi $3.2 \mathrm{ml}, 1.6$ molar, and diethylamine $0.10 \mathrm{ml}, 0.96 \mathrm{mmol}$ ).Yield: $324 \mathrm{mg}$ (61\%); m.p. $265-267^{\circ} \mathrm{C}$ (yellow prism). Recovered starting acid: $27 \mathrm{mg}(9 \%)$. IR (KBr): $v_{\max }=3035,1646$, 1463, 1429, 1300, 1217, 1098, 1033, 872, 836, $811 \mathrm{~cm}^{-1} .{ }^{1} \mathrm{H}$ NMR $\left(\mathrm{CDCl}_{3}, 300 \mathrm{MHz}\right): \delta=9.38$ (s, $1 \mathrm{H}, \mathrm{NH}$ ), 8.92 (d, $1 \mathrm{H}, J=5.1 \mathrm{~Hz}, \mathrm{~N}=\mathrm{C} \underline{\mathrm{H}}-\mathrm{CH}=\mathrm{CH}), 8.63$ (d, $1 \mathrm{H}, J=8.2 \mathrm{~Hz}, \mathrm{~N}=\mathrm{CH}-\mathrm{CH}=\mathrm{C} \underline{\mathrm{H}}$ ), 7.66 (d, 2H, $J=8.2 \mathrm{~Hz}, 2 \mathrm{CHAr}$ ), 7.38 (dd, $1 \mathrm{H}, J=8.2$ and $5.1 \mathrm{~Hz}, \mathrm{~N}=\mathrm{CH}-\mathrm{C} \underline{\mathrm{H}}=\mathrm{CH}$ ), 7.35 (d, 2H, $J=8.2 \mathrm{~Hz}, 2 \mathrm{CHAr}), 7.03$ (s, $1 \mathrm{H}, \mathrm{C} \underline{\mathrm{H}}=\mathrm{C}-\mathrm{NH}), 2.43\left(\mathrm{~s}, 1 \mathrm{H}, \mathrm{CH}_{3}\right) .{ }^{13} \mathrm{C} \mathrm{NMR}\left(\mathrm{CDCl}_{3}, 75 \mathrm{MHz}\right) \delta=$ 163.9 (C=O), 155.1 ( $\mathrm{N}=\underline{\mathrm{C}} \mathrm{H}-\mathrm{CH}=\mathrm{CH}), 154.9$ ( $\mathrm{C}=\mathrm{C}-\mathrm{CO}), 152.1$ (CH=ㅡ-NH), $143.4(\mathrm{CH}=\underline{\mathrm{C}}-\mathrm{NH})$, 140.5 ( $\left.\underline{\mathrm{CAr}}-\mathrm{CH}_{3}\right), 135.8$ ( $\left.\mathrm{N}=\mathrm{CH}-\mathrm{CH}=\underline{\mathrm{C} H}\right), 130.8$ ( $\underline{\mathrm{CAr}}$ ), 130.0 (2 CHAr), 126.2 (2 CHAr), 121.3 $(\mathrm{N}=\mathrm{CH}-\underline{\mathrm{CH}}=\mathrm{CH}), 120.2$ (C=-ㅡ-CO), $105.5(\underline{\mathrm{CH}}=\mathrm{C}-\mathrm{NH}), 21.3\left(\underline{\mathrm{CH}}_{3}\right) . \mathrm{MS}(\mathrm{EI}): \mathrm{m} / \mathrm{z}(\%)=236$ $\left(\mathrm{M}^{+}, 100\right), 221\left(\mathrm{M}^{+}-\mathrm{CH}_{3}, 3\right), 207\left(\mathrm{M}^{+}-\mathrm{COH}, 5\right), 180\left(\mathrm{M}^{+}-\mathrm{C}_{2} \mathrm{H}_{2} \mathrm{NO}, 4\right), 119\left(\mathrm{M}^{+}-\mathrm{C}_{8} \mathrm{H}_{7} \mathrm{~N}, 3\right), 91$ $\left(\mathrm{C}_{7} \mathrm{H}_{7}{ }^{+}, 3\right)$. HRMS: $\mathrm{m} / z$ calcd for $\mathrm{C}_{15} \mathrm{H}_{12} \mathrm{~N}_{2} \mathrm{O}\left(\mathrm{M}^{+}\right), 236.0950$, found 236.0954 .

7-(4-Chlorophenyl)-5,6-dihydro[1,6]naphthyridin-5-one (8d). From 2-methylnicotinic acid 4 (307 mg, $2.25 \mathrm{mmol}$ ) and p-chlorobenzonitrile d (313 mg, $2.25 \mathrm{mmol}$ ) and lithium diisopropylamide (from BuLi $3.2 \mathrm{ml}, 1.6 \mathrm{molar}$, and isopropylamine $0.5 \mathrm{ml}, 5 \mathrm{mmol}$ ).Yield: $432 \mathrm{mg}$ (75\%); m.p. 310-312 ${ }^{\circ} \mathrm{C}$ dec. (yellow solid). Recovered starting acid: $24 \mathrm{mg}$ (8\%). IR $(\mathrm{KBr}): v_{\max }=3163,2964,1674,1628,1589,1404,1300,1261,1192,1093,871,805,774 \mathrm{~cm}^{-1}$. ${ }^{1} \mathrm{H}$ NMR (DMSO, 400MHz): $\delta=9.14(\mathrm{~s}, 1 \mathrm{H}, \mathrm{NH}), 8.90(\mathrm{~d}, 1 \mathrm{H}, J=5.1 \mathrm{~Hz}, \mathrm{~N}=\mathrm{C} \underline{\mathrm{H}}-\mathrm{CH}=\mathrm{CH}$ ), 8.49 (d, $1 \mathrm{H}, J=8.2 \mathrm{~Hz}, \mathrm{~N}=\mathrm{CH}-\mathrm{CH}=\mathrm{CH}), 7.83$ (d, 2H, $J=8.2 \mathrm{~Hz}, 2 \mathrm{CHAr}), 7.54$ (d, 2H, $J=$ $8.2 \mathrm{~Hz}, 2 \mathrm{CHAr}$ ), 7.46 (dd, $1 \mathrm{H}, J=8.2$ and $5.1 \mathrm{~Hz}, \mathrm{~N}=\mathrm{CH}-\mathrm{C} \underline{\mathrm{H}}=\mathrm{CH}$ ), 6.90 (s, $1 \mathrm{H}, \mathrm{C} \underline{\mathrm{H}}=\mathrm{C}-\mathrm{NH}$ ), $2.43\left(\mathrm{~s}, 1 \mathrm{H}, \mathrm{CH}_{3}\right) .{ }^{13} \mathrm{C}$ NMR (DMSO, 75MHz) $\delta=163.5(\mathrm{C}=\mathrm{O}), 155.6(\mathrm{~N}=\mathrm{C} H-\mathrm{CH}=\mathrm{CH}), 154.9$ ( $\underline{\mathrm{C}}=\mathrm{C}-\mathrm{CO}), 143.6(\mathrm{CH}=\underline{\mathrm{C}}-\mathrm{NH}), 135.6$ ( $\mathrm{N}=\mathrm{CH}-\mathrm{CH}=\underline{\mathrm{CH}}), 130.4$ (CAr), 129.5 (2 CHAr), 129.45 (2 CHAr), 128.5 (ClCAr), 122.4 ( $\mathrm{N}=\mathrm{CH}-\underline{\mathrm{CH}}=\mathrm{CH}), 122.0$ (C=ㅡ-CO), 105.6 ( $\underline{\mathrm{CH}}=\mathrm{C}-\mathrm{NH}) . \mathrm{MS}$ (EI): $\mathrm{m} / \mathrm{z}(\%)=258\left({ }^{37} \mathrm{ClM}^{+}, 34\right), 256\left({ }^{35} \mathrm{ClM}^{+}, 100\right), 221\left(\mathrm{M}^{+}-\mathrm{Cl}, 7\right), 193\left(\mathrm{M}^{+}-\mathrm{CO}-\mathrm{Cl}, 8\right), 91\left(\mathrm{C}_{7} \mathrm{H}_{7}{ }^{+}\right.$, 4). HRMS: $\mathrm{m} / \mathrm{z}$ calcd for $\mathrm{C}_{14} \mathrm{H}_{9} \mathrm{~N}_{2} \mathrm{O}^{37} \mathrm{Cl}\left(\mathrm{M}^{+}\right)$258.0374, found: 258.0381. $\mathrm{m} / \mathrm{z}$ calcd for $\mathrm{C}_{14} \mathrm{H}_{9} \mathrm{~N}_{2} \mathrm{O}^{35} \mathrm{Cl}\left(\mathrm{M}^{+}\right)$, 256.0403, found: 256.0412.

7-(2-Pyridinyl)-5,6-dihydro[1,6]naphthyridin-5-one (8e). From 2-methylnicotinic acid 4 (307 mg, $2.25 \mathrm{mmol}$ ) and 2-cyanopyridine e (237 mg, $2.25 \mathrm{mmol}$ ) and lithium diisopropylamide (from BuLi $3.2 \mathrm{ml}, 1.6$ molar, and isopropylamine $0.14 \mathrm{ml}, 0.96 \mathrm{mmol}$ ). Yield: $264 \mathrm{mg}$ (56\%). IR (KBr): $v_{\max }=3321,3050-2900,1680,1630,1590,1531,1469,998,778 \mathrm{~cm}^{-1} .{ }^{1} \mathrm{H}$ NMR $\left(\mathrm{CDCl}_{3}, 500 \mathrm{MHz}\right): \delta=9.30(\mathrm{~s}, 1 \mathrm{H}, \mathrm{NH}), 8.91(\mathrm{~m}, 1 \mathrm{H}, \mathrm{C} \underline{\mathrm{H}}-\mathrm{N}-\mathrm{C}-\mathrm{C}-\mathrm{CONH}), 8.65(\mathrm{~m}, 1 \mathrm{H}, \mathrm{C} \underline{\mathrm{H}}-\mathrm{N}-$ C-C-NHCO), 8.55 (m, 1H, NHCO-C-Cㅡㅡ), 8.18 (m, 1H, N-CH-CH-CH-Cㅡㅡ), 7.95 (m, 1H, N-

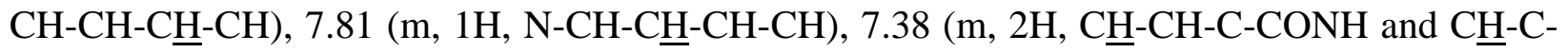
$\mathrm{NH}) .{ }^{13} \mathrm{C}$ NMR (MeOD, 75MHz) $\delta=162.4(\mathrm{C}=\mathrm{O}), 155.1$ (CH-N-C-C-CONH), 155.0 (ㅡ-CCONH), 149.3 (ㅡH-N-C-C-NHCO), 148.0 (ㅡ-C-CONH), 139.4 (ㅡ-C-NHCO), 137.0 (N-CH-

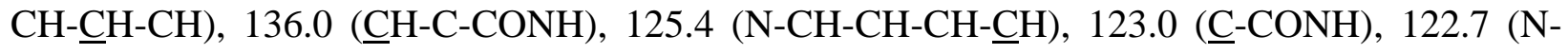

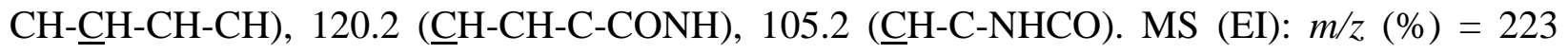


( $\left.\mathrm{M}^{+}, 100\right), 195\left(\mathrm{M}^{+}-\mathrm{CO}, 18\right), 119\left(\mathrm{C}_{8} \mathrm{H}_{7} \mathrm{O}^{+}, 8\right), 79\left(\mathrm{C}_{5} \mathrm{H}_{5} \mathrm{~N}^{+}, 12\right), 78\left(\mathrm{C}_{5} \mathrm{H}_{4} \mathrm{~N}^{+}, 11\right)$. HRMS: $\mathrm{m} / \mathrm{z}$ calcd for $\mathrm{C}_{13} \mathrm{H}_{9} \mathrm{~N}_{3} \mathrm{O}\left(\mathrm{M}^{+}\right)$223.0746, found: 223.0745 .

2,4,6-Tri(2-pyridyl)-1,3,5-triazine (9). From 3-methyl-2-thiophenecarboxylic acid 3 (326 mg, $2.25 \mathrm{mmol}$ ) and 2-cyanopyridine e (235 $\mathrm{mg}, 2.25 \mathrm{mmol}$ ) and lithium diethylamide (from BuLi $3.2 \mathrm{ml}, 1.6$ molar, and diethylamine $0.5 \mathrm{ml}, 5 \mathrm{mmol}$ ). Yield: $51 \mathrm{mg}$ (46\%); m.p. $180-81^{\circ} \mathrm{C}$ (dec.) (yellow prism). Recovered starting acid: $212 \mathrm{mg}$ (65\%). IR (KBr): $v_{\max }=3500-2800,1642,1582$, 1529, 1368, 1253, 995, $769 \mathrm{~cm}^{-1} .{ }^{1} \mathrm{H}$ NMR $\left(\mathrm{CDCl}_{3}, 400 \mathrm{MHz}\right): \delta=8.96$ (ddd, $1 \mathrm{H}, J=4.8,2.0$, $1.6 \mathrm{~Hz}, \mathrm{~N}-\mathrm{CH}$ ), 8.86 (dt, $1 \mathrm{H}, J=8.0$ and $1.2 \mathrm{~Hz}, \mathrm{~N}-\mathrm{CH}-\mathrm{CH}-\mathrm{CH}-\mathrm{C}$ ), 7.97 (dt, $1 \mathrm{H}, J=7.6$ and $2.0 \mathrm{~Hz}, \mathrm{~N}-\mathrm{CH}-\mathrm{CH}-\mathrm{C} \underline{\mathrm{H}}-\mathrm{CH}$ ), 7.55 (ddd, $1 \mathrm{H}, J=7.6,4.8$ and $1.6 \mathrm{~Hz}, \mathrm{~N}-\mathrm{CH}-\mathrm{C}-\mathrm{H}-\mathrm{CH}-\mathrm{CH}$ ). ${ }^{13} \mathrm{C}$ NMR $\left(\mathrm{CDCl}_{3}, 75 \mathrm{MHz}\right) \delta=161.3(\mathrm{~N}-\mathrm{C}=\mathrm{N}), 150.7(\mathrm{~N}-\mathrm{CH}), 137.3(\mathrm{~N}-\mathrm{CH}-\mathrm{CH}-\underline{\mathrm{CH}}-\mathrm{CH}), 128.9(\mathrm{C}-$ $\mathrm{N}-\mathrm{CH}), 126.8$ (N-CH-Ch-CH-CH), $125.4(\mathrm{~N}-\mathrm{CH}-\mathrm{CH}-\mathrm{CH}-\underline{\mathrm{CH}}) . \mathrm{MS}(\mathrm{EI}): \mathrm{m} / \mathrm{z}(\%)=313\left(\mathrm{M}^{+}+1\right.$, 22), $312\left(\mathrm{M}^{+}, 100\right), 208\left(\mathrm{M}^{+}-\mathrm{C}_{6} \mathrm{H}_{4} \mathrm{~N}_{2}, 56\right), 105\left(\mathrm{C}_{6} \mathrm{H}_{5} \mathrm{~N}_{2}{ }^{+}, 57\right), 104\left(\mathrm{M}^{+}-2 \mathrm{C}_{6} \mathrm{H}_{4} \mathrm{~N}_{2}, 26\right), 78$ $\left(\mathrm{C}_{5} \mathrm{H}_{4} \mathrm{~N}^{+}\right.$, 14). HRMS: $\mathrm{m} / \mathrm{z}$ calcd for $\mathrm{C}_{18} \mathrm{H}_{12} \mathrm{~N}_{6}\left(\mathrm{M}^{+}\right)$312.1114, found: 312.1115 .

\section{Acknowledgements}

The present research has been financed by DCICYT (PB-98-1430-C02-01). One of us (E.M.B.) acknowledges a grant by DGICYT.

\section{References}

1. (a) Schultz, A. G. Chem. Rev. 1973, 73, 385. (b) Curran, D. P.; Liu, H. J. Am. Chem. Soc. 1992, 114, 5863. (c) Williams, D. R.; Lowder, P. D.; Gu, Y-G. Tetrahedron Lett. 1997, 38, 327. (d) Kozikowski, A. P.; Campiani, G.; Sun, L-Q.; Wang, S.; Saxena, A.; Doctor, B. P. J. Am. Chem. Soc. 1996, 118, 11357. (e) Brickner, S. J. Chemistry and Industry 1997, 131.

2. (a) Murray, T. J.; Zimmerman, S. C. Tetrahedron Lett. 1995, 36, 7627. (b) Lee, L. F.; Normansell, J. E. J. Org. Chem. 1990, 55, 2964.

3. Boga, C.; Corradi Bonamartini, A.; Forlani, L.; Modarell, V.; Righi, L.; Sgarabotto, P.; Todesco, P. E. Eur. J. Org. Chem. 2001, 1175.

4. Aakeröy, C. B.; Beatty, A. M.; Nieuwenhuyzen, M.; Zou, M. Tetrahedron 2000, 56, 6693.

5. (a) Meislich, H. Pyridine and its derivatives, Klingsberg, E. Ed; Interscience: New York, 1962; Vol. 3, p 509. (b) Jones, G. In Comprehensive Heterocyclic Chemistry II; Katritzky, A. R.; Rees, C. W.; Scriven, E. F.V., Eds; Pergamon Press: Oxford, 1996, Vol. 2; p 395. (c) Wenner, W.; Plati, J. T. J. Chem. Soc. 1946, 751.

6. (a) Jain, R.; Roschangar, F.; Ciufolini, M. A. Tetrahedron Lett. 1995, 36, 3307. (b) Tohda, Y.; Yanagidani, T.; Hiramatsu, S-i.; Nishiwaki, N.; Tani, K.; Ariga, M. Bull. Chem. Soc. Jpn. 1997, 70, 2781. (c) Mekouar, K.; Genisson, V.; Leue, S.; Greene, A.E. J. Org. Chem, 2000, 65, 5212. 
7. Kelly, T. R.; Bell, S. H.; Ohashi, N.; Armstrong-Chong, R. J. J. Am. Chem. Soc. 1988, 110, 6471.

8. (a) Brun, E. M.; Gil, S.; Mestres, R.; Parra, M. Synlett. 1999, 1088. (b) Brun, E. M.; Gil, S.; Mestres, R.; Parra, M. Synthesis 2000, 273.

9. Chen, Y.; Li, T.; Sieburth, S. McN. J. Org. Chem. 2001, 66, 6826.

10. (a) Guion, T. S.; Koller, M. U.; Lachicotte, R. J.; Rutledge, R. N.; Hildebran, K. C.; Le, P. H.; Bearn, C.F. Synth. Commun. 1996, 26, 1753. (b) Julia, M.; Pfeuty-Saint Jalmes, V.; Plé, K.; Verpeaux, J-N.; Hollingworth, G. Bull. Soc. Chim. Fr. 1996, 133, 15. (c) Belletire, J. L.; Speletzer, E. G. Synth. Commun. 1986, 16, 575

11. (a) Gould, N. P.; Lee, T-J. J. Org. Chem. 1980, 45, 4528. (b) Epsztajn, J.; Poltka, M.W.; Scianowski, J. Synth. Commun. 1992, 22, 1239

12. Brun, E. M.; Casades, I.; Gil, S.; Mestres, R.; Parra, M. Tetrahedron Lett. 1998, 39, 5443. 